\title{
Benchmarks for Electronically Excited States with CASSCF Methods
}

Supporting Information

Benjamin Helmich-Paris

June 6, 2019 


\section{States included in the state-averaged CASSCF calculations with TZVP basis set.}

Table 1: States included in the state-averaged CASSCF calculations with TZVP basis set. Only symmetry up to $\mathrm{D}_{2 \mathrm{~h}}$ and subgroups were exploited in the calculation.

\begin{tabular}{|c|c|c|c|}
\hline Molecule & CAS & Irrep & Count \\
\hline ethene $\left(D_{2 h}\right)$ & $(2,2)$ & $\begin{array}{l}\mathrm{A}_{\mathrm{g}} \\
\mathrm{B}_{1 \mathrm{u}}\end{array}$ & $\begin{array}{l}1 \\
1\end{array}$ \\
\hline E-butadiene $\left(\mathrm{C}_{2 \mathrm{~h}}\right)$ & $(4,4)$ & $\begin{array}{l}\mathrm{A}_{\mathrm{g}} \\
\mathrm{B}_{1 \mathrm{u}}\end{array}$ & $\begin{array}{r}2 \\
1^{\mathrm{a}}\end{array}$ \\
\hline all-E-hexatriene $\left(\mathrm{C}_{2 \mathrm{~h}}\right)$ & $(6,6)$ & $\begin{array}{l}\mathrm{A}_{\mathrm{g}} \\
\mathrm{B}_{1 \mathrm{u}}\end{array}$ & $\begin{array}{r}2 \\
1^{\mathrm{a}}\end{array}$ \\
\hline all-E-octatetraene $\left(\mathrm{C}_{2 \mathrm{~h}}\right)$ & $(8,8)$ & $\begin{array}{l}\mathrm{A}_{\mathrm{g}} \\
\mathrm{B}_{1 \mathrm{u}}\end{array}$ & $\begin{array}{r}2 \\
1^{\mathrm{a}}\end{array}$ \\
\hline cyclopropene $\left(\mathrm{C}_{2 \mathrm{v}}\right)$ & $(4,3)$ & $\begin{array}{l}\mathrm{A}_{1} \\
\mathrm{~B}_{1} \\
\mathrm{~B}_{2}\end{array}$ & $\begin{array}{l}1 \\
1 \\
1\end{array}$ \\
\hline cyclopentadiene $\left(\mathrm{C}_{2 \mathrm{v}}\right)$ & $(4,4)$ & $\begin{array}{l}A_{1} \\
B_{2}\end{array}$ & $\begin{array}{l}3 \\
1\end{array}$ \\
\hline norbornadiene $\left(\mathrm{C}_{2 \mathrm{v}}\right)$ & $(4,4)$ & $\begin{array}{l}\mathrm{A}_{1} \\
\mathrm{~A}_{2} \\
\mathrm{~B}_{2}\end{array}$ & $\begin{array}{l}1 \\
2 \\
2\end{array}$ \\
\hline benzene $\left(D_{2 h}\right)$ & $(6,6)$ & $\begin{array}{l}\mathrm{A}_{\mathrm{g}} \\
\mathrm{B}_{1 \mathrm{~g}} \\
\mathrm{~B}_{2 \mathrm{u}} \\
\mathrm{B}_{3 \mathrm{u}}\end{array}$ & $\begin{array}{l}2 \\
1 \\
2 \\
2\end{array}$ \\
\hline naphthalene $\left(D_{2 h}\right)$ & $(10,10)$ & $\begin{array}{l}\mathrm{A}_{\mathrm{g}} \\
\mathrm{B}_{1 \mathrm{~g}} \\
\mathrm{~B}_{1 \mathrm{u}} \\
\mathrm{B}_{2 \mathrm{u}}\end{array}$ & $\begin{array}{l}3 \\
2 \\
5 \\
3\end{array}$ \\
\hline furan $\left(\mathrm{C}_{2 \mathrm{v}}\right)$ & $(6,5)$ & $\begin{array}{l}\mathrm{A}_{1} \\
\mathrm{~B}_{2}\end{array}$ & $\begin{array}{l}3 \\
1\end{array}$ \\
\hline pyrrole $\left(\mathrm{C}_{2 \mathrm{v}}\right)$ & $(6,5)$ & $\begin{array}{l}\mathrm{A}_{1} \\
\mathrm{~B}_{2}\end{array}$ & $\begin{array}{l}3 \\
1\end{array}$ \\
\hline imidazole $\left(\mathrm{C}_{\mathrm{s}}\right)$ & $(8,7)$ & $\begin{array}{l}A^{\prime} \\
A^{\prime \prime}\end{array}$ & $\begin{array}{l}5 \\
2\end{array}$ \\
\hline pyridine $\left(\mathrm{C}_{2 \mathrm{v}}\right)$ & $(8,7)$ & $\mathrm{A}_{1}$ & 4 \\
\hline
\end{tabular}




\begin{tabular}{|c|c|c|c|}
\hline Molecule & CAS & Irrep & Count \\
\hline & & $\mathrm{A}_{2}$ & 1 \\
\hline & & $\mathrm{B}_{1}$ & 1 \\
\hline & & $\mathrm{B}_{2}$ & 3 \\
\hline \multirow[t]{8}{*}{ pyrazine $\left(D_{2 h}\right)$} & $(10,8)$ & $\mathrm{A}_{\mathrm{g}}$ & 2 \\
\hline & & $B_{1 g}$ & 1 \\
\hline & & $B_{2 g}$ & 1 \\
\hline & & $\mathrm{B}_{3 \mathrm{~g}}$ & 1 \\
\hline & & $\mathrm{A}_{\mathrm{u}}$ & 2 \\
\hline & & $\mathrm{B}_{1 \mathrm{u}}$ & 1 \\
\hline & & $\mathrm{B}_{2 \mathrm{u}}$ & 2 \\
\hline & & $\mathrm{B}_{3 \mathrm{u}}$ & 2 \\
\hline \multirow{4}{*}{ pyrimidine $\left(\mathrm{C}_{2 \mathrm{v}}\right)$} & $(12,9)$ & $\mathrm{A}_{1}$ & 4 \\
\hline & & $\mathrm{A}_{2}$ & 1 \\
\hline & & $\mathrm{B}_{1}$ & 1 \\
\hline & & $\mathrm{B}_{2}$ & 3 \\
\hline \multirow[t]{4}{*}{ pyridazine $\left(\mathrm{C}_{2 \mathrm{v}}\right)$} & $(12,9)$ & $\mathrm{A}_{1}$ & 5 \\
\hline & & $\mathrm{A}_{2}$ & 2 \\
\hline & & $\mathrm{B}_{1}$ & 2 \\
\hline & & $\mathrm{B}_{2}$ & 4 \\
\hline \multirow[t]{4}{*}{$s$-triazine $\left(\mathrm{C}_{2 \mathrm{v}}\right)$} & $(12,9)$ & $\mathrm{A}_{1}$ & 5 \\
\hline & & $\mathrm{A}_{2}$ & 4 \\
\hline & & $\mathrm{B}_{1}$ & 4 \\
\hline & & $\mathrm{B}_{2}$ & 4 \\
\hline \multirow[t]{8}{*}{$s$-tetrazine $\left(\mathrm{D}_{2 \mathrm{~h}}\right)$} & $(14,10)$ & $\mathrm{A}_{\mathrm{g}}$ & 1 \\
\hline & & $\mathrm{B}_{1 \mathrm{~g}}$ & 2 \\
\hline & & $\mathrm{B}_{2 \mathrm{~g}}$ & 2 \\
\hline & & $\mathrm{B}_{3 \mathrm{~g}}$ & 3 \\
\hline & & $\mathrm{A}_{\mathrm{u}}$ & 2 \\
\hline & & $\mathrm{B}_{1 \mathrm{u}}$ & 2 \\
\hline & & $\mathrm{B}_{2 \mathrm{u}}$ & 3 \\
\hline & & $\mathrm{B}_{3 \mathrm{u}}$ & 3 \\
\hline \multirow[t]{3}{*}{ formaldehyde $\left(\mathrm{C}_{2 \mathrm{v}}\right)$} & $(6,4)$ & $\mathrm{A}_{1}$ & 2 \\
\hline & & $\mathrm{A}_{2}$ & 1 \\
\hline & & $\mathrm{B}_{1}$ & 1 \\
\hline \multirow[t]{3}{*}{ acetone $\left(\mathrm{C}_{2 \mathrm{v}}\right)$} & $(6,5)$ & $\mathrm{A}_{1}$ & 3 \\
\hline & & $\mathrm{A}_{2}$ & 1 \\
\hline & & $\mathrm{B}_{1}$ & 1 \\
\hline \multirow[t]{2}{*}{$p$-benzoquinone $\left(\mathrm{D}_{2 \mathrm{~h}}\right)$} & $(12,10)$ & $\mathrm{A}_{\mathrm{g}}$ & 3 \\
\hline & & $\mathrm{B}_{1 \mathrm{~g}}$ & 2 \\
\hline
\end{tabular}




\begin{tabular}{|c|c|c|c|}
\hline Molecule & CAS & Irrep & Count \\
\hline & & $B_{2 g}$ & 2 \\
\hline & & $\mathrm{B}_{3 \mathrm{~g}}$ & 2 \\
\hline & & $\mathrm{A}_{\mathrm{u}}$ & 2 \\
\hline & & $\mathrm{B}_{1 \mathrm{u}}$ & 2 \\
\hline & & $\mathrm{B}_{2 \mathrm{u}}$ & 1 \\
\hline & & $\mathrm{B}_{3 \mathrm{u}}$ & 1 \\
\hline \multirow[t]{2}{*}{ formamide $\left(\mathrm{C}_{\mathrm{s}}\right)$} & $(6,4)$ & $A^{\prime}$ & 3 \\
\hline & & A" & 1 \\
\hline \multirow{2}{*}{ acetamide $\left(\mathrm{C}_{\mathrm{s}}\right)$} & $(6,4)$ & $A^{\prime}$ & 3 \\
\hline & & A" & 1 \\
\hline \multirow[t]{2}{*}{ propanamide $\left(\mathrm{C}_{\mathrm{s}}\right)$} & $(6,4)$ & $A^{\prime}$ & 3 \\
\hline & & A" & 1 \\
\hline \multirow[t]{2}{*}{ cytosine $\left(\mathrm{C}_{\mathrm{s}}\right)$} & $(14,11)$ & $A^{\prime}$ & 5 \\
\hline & & A" & 2 \\
\hline \multirow[t]{2}{*}{ thymine $\left(\mathrm{C}_{\mathrm{s}}\right)$} & $(16,12)$ & $A^{\prime}$ & 6 \\
\hline & & A" & 4 \\
\hline \multirow[t]{2}{*}{$\operatorname{uracil}\left(\mathrm{C}_{\mathrm{s}}\right)$} & $(14,10)$ & $A^{\prime}$ & 5 \\
\hline & & A" & 4 \\
\hline \multirow[t]{2}{*}{ adenine $\left(\mathrm{C}_{\mathrm{s}}\right)$} & $(18,13)$ & $A^{\prime}$ & 5 \\
\hline & & A" & 5 \\
\hline
\end{tabular}

\footnotetext{
${ }^{\mathrm{a}}$ We averaged over two states but the weight of first state was set to 0 .
} 


\section{States included in the state-averaged CASSCF calculations with aug-cc-pVTZ basis set.}

Table 2: States included in the state-averaged CASSCF calculations with aug-cc-pVTZ basis set. Only symmetry up to $\mathrm{D}_{2 \mathrm{~h}}$ and subgroups were exploited in the calculation.

\begin{tabular}{lrlr}
\hline \multicolumn{1}{c}{ Molecule } & CAS & Irrep & Count \\
\hline ethene $\left(\mathrm{D}_{2 \mathrm{~h}}\right)$ & $(2,2)$ & $\mathrm{A}_{\mathrm{g}}$ & 1 \\
& & $\mathrm{~B}_{1 \mathrm{u}}$ & 1 \\
E-butadiene $\left(\mathrm{C}_{2 \mathrm{~h}}\right)$ & $(4,4)$ & $\mathrm{A}_{\mathrm{g}}$ & 2 \\
cyclopropene $\left(\mathrm{C}_{2 \mathrm{v}}\right)$ & $(4,3)$ & $\mathrm{A}_{1}$ & 1 \\
& & $\mathrm{~B}_{1}$ & 1 \\
& & $\mathrm{~B}_{2}$ & 1 \\
benzene $\left(\mathrm{C}_{1}\right)$ & $(6,6)$ & $\mathrm{A}_{1}$ & 7 \\
s-tetrazine $\left(\mathrm{C}_{1}\right)$ & $(14,10)$ & $\mathrm{A}_{1}$ & 32 \\
formaldehyde $\left(\mathrm{C}_{2 \mathrm{v}}\right)$ & $(6,4)$ & $\mathrm{A}_{1}$ & 2 \\
& & $\mathrm{~A}_{2}$ & 1 \\
& & $\mathrm{~B}_{1}$ & 1 \\
acetone $\left(\mathrm{C}_{1}\right)$ & $(6,4)$ & $\mathrm{A}_{1}$ & 4 \\
formamide $\left(\mathrm{C}_{\mathrm{s}}\right)$ & $(6,4)$ & $\mathrm{A}^{\prime}$ & 3 \\
& & $\mathrm{~A}^{\prime \prime}$ & 1 \\
\hline
\end{tabular}


3 Vertical excitation energies with TZVP basis set

Table 3: Vertical singlet excitation energies in eV with TZVP basis set.

\begin{tabular}{|c|c|c|c|c|c|c|c|}
\hline Molecule & State & Type & MC-RPA & MC-TDA & SA-CAS & $\mathrm{CC}^{\mathrm{a}, \mathrm{b}}\left(\% \mathrm{~T}_{1}\right)$ & $\mathrm{CASPT2}^{\mathrm{a}, \mathrm{c}}$ \\
\hline ethene & $1{ }^{1} \mathrm{~B}_{1 \mathrm{u}}$ & $\pi \rightarrow \pi^{*}$ & 8.76 & 9.06 & 9.17 & $8.37(96.9)$ & 8.62 \\
\hline \multirow[t]{2}{*}{ E-butadiene } & $1{ }^{1} \mathrm{~B}_{\mathrm{u}}$ & $\pi \rightarrow \pi^{*}$ & 7.39 & 7.63 & 8.30 & $6.58(93.7)$ & 6.47 \\
\hline & $2{ }^{1} \mathrm{~A}_{\mathrm{g}}$ & $\pi \rightarrow \pi^{*}$ & 6.97 & 7.10 & 6.59 & $6.77(72.8)$ & 6.83 \\
\hline \multirow{2}{*}{$\begin{array}{l}\text { all-E- } \\
\text { hexatriene }\end{array}$} & $1{ }^{1} \mathrm{~B}_{\mathrm{u}}$ & $\pi \rightarrow \pi^{*}$ & 6.57 & 6.78 & 7.33 & $5.58(92.6)$ & 5.31 \\
\hline & $2{ }^{1} \mathrm{~A}_{\mathrm{g}}$ & $\pi \rightarrow \pi^{*}$ & 5.87 & 5.98 & 5.52 & $5.72(65.8)$ & 5.42 \\
\hline \multirow{2}{*}{$\begin{array}{l}\text { all-E- } \\
\text { octatetraene }\end{array}$} & $2{ }^{1} \mathrm{~A}_{\mathrm{g}}$ & $\pi \rightarrow \pi^{*}$ & 5.09 & 5.19 & 4.77 & $4.97(62.9)$ & 4.64 \\
\hline & $1{ }^{1} \mathrm{~B}_{\mathrm{u}}$ & $\pi \rightarrow \pi^{*}$ & 6.04 & 6.22 & 6.69 & 4.94 (91.9) & 4.70 \\
\hline \multirow[t]{2}{*}{ cyclopropene } & ${ }^{1}{ }^{1} \mathrm{~B}_{1}$ & $\sigma \rightarrow \pi^{*}$ & 7.57 & 7.67 & 7.09 & $6.90(93.0)$ & 6.76 \\
\hline & $1^{1} \mathrm{~B}_{2}$ & $\pi \rightarrow \pi^{*}$ & 7.69 & 7.98 & 8.20 & $7.10(95.5)$ & 7.06 \\
\hline \multirow[t]{3}{*}{ cyclopentadiene } & $1^{1} \mathrm{~B}_{2}$ & $\pi \rightarrow \pi^{*}$ & 6.61 & 6.86 & 7.37 & $5.73(94.3)$ & 5.51 \\
\hline & $2{ }^{1} \mathrm{~A}_{1}$ & $\pi \rightarrow \pi^{*}$ & 6.87 & 7.02 & 6.54 & $6.61(79.3)$ & 6.31 \\
\hline & $3{ }^{1} \mathrm{~A}_{1}$ & $\pi \rightarrow \pi^{*}$ & 9.26 & 9.59 & 10.78 & $8.69(93.1)$ & 8.52 \\
\hline \multirow[t]{4}{*}{ norbornadiene } & $1^{1} \mathrm{~A}_{2}$ & $\pi \rightarrow \pi^{*}$ & 6.78 & 7.01 & 6.63 & $5.64(93.4)$ & 5.34 \\
\hline & $1^{1} \mathrm{~B}_{2}$ & $\pi \rightarrow \pi^{*}$ & 7.88 & 8.05 & 8.01 & $6.49(91.9)$ & 6.11 \\
\hline & $2{ }^{1} \mathrm{~B}_{2}$ & $\pi \rightarrow \pi^{*}$ & 8.87 & 9.04 & 9.33 & $7.64(93.8)$ & 7.32 \\
\hline & $2{ }^{1} \mathrm{~A}_{2}$ & $\pi \rightarrow \pi^{*}$ & 8.71 & 8.91 & 9.14 & $7.71(93.0)$ & 7.44 \\
\hline \multirow[t]{4}{*}{ benzene } & $1^{1} \mathrm{~B}_{2 \mathrm{u}}$ & $\pi \rightarrow \pi^{*}$ & 4.65 & 4.81 & 4.81 & $5.07(85.8)$ & 5.05 \\
\hline & $1{ }^{1} \mathrm{~B}_{1 \mathrm{u}}$ & $\pi \rightarrow \pi^{*}$ & 7.25 & 7.49 & 7.96 & $6.68(93.6)$ & 6.45 \\
\hline & $1{ }^{1} \mathrm{E}_{1 \mathrm{u}}$ & $\pi \rightarrow \pi^{*}$ & 8.11 & 8.38 & 9.30 & $7.45(92.2)$ & 7.07 \\
\hline & $2{ }^{1} \mathrm{E}_{2 \mathrm{~g}}$ & $\pi \rightarrow \pi^{*}$ & 8.08 & 8.33 & 8.00 & $8.43(65.6)$ & 8.21 \\
\hline \multirow[t]{8}{*}{ naphthalene ${ }^{\mathrm{d}}$} & ${ }^{1}{ }^{1} \mathrm{~B}_{2 \mathrm{u}}$ & $\pi \rightarrow \pi^{*}$ & 3.99 & 4.11 & 4.10 & $4.27(85.2)$ & 4.24 \\
\hline & ${ }^{1}{ }^{1} \mathrm{~B}_{1 \mathrm{u}}$ & $\pi \rightarrow \pi^{*}$ & 5.97 & 6.15 & 6.48 & $5.98(90.6)$ & 4.77 \\
\hline & $2{ }^{1} \mathrm{~A}_{\mathrm{g}}$ & $\pi \rightarrow \pi^{*}$ & 5.59 & 5.73 & 5.71 & $5.03(82.2)$ & 5.90 \\
\hline & $1{ }^{1} \mathrm{~B}_{3 \mathrm{~g}}$ & $\pi \rightarrow \pi^{*}$ & 6.50 & 6.58 & 6.37 & $6.07(79.6)$ & 6.00 \\
\hline & $2{ }^{1} \mathrm{~B}_{2 \mathrm{u}}$ & $\pi \rightarrow \pi^{*}$ & 7.12 & 7.33 & 8.09 & $6.33(90.7)$ & 6.07 \\
\hline & $2{ }^{1} \mathrm{~B}_{3 \mathrm{~g}}$ & $\pi \rightarrow \pi^{*}$ & 7.41 & 7.79 & 8.44 & $6.79(91.3)$ & 6.48 \\
\hline & $2{ }^{1} \mathrm{~B}_{1 \mathrm{u}}$ & $\pi \rightarrow \pi^{*}$ & 7.25 & 7.49 & 7.45 & $6.57(90.5)$ & 6.33 \\
\hline & $3{ }^{1} \mathrm{~A}_{\mathrm{g}}$ & $\pi \rightarrow \pi^{*}$ & 6.86 & 7.01 & 6.73 & $6.90(70.0)$ & 6.71 \\
\hline \multirow[t]{3}{*}{ furan } & $1^{1} \mathrm{~B}_{2}$ & $\pi \rightarrow \pi^{*}$ & 7.20 & 7.47 & 7.79 & $6.60(92.6)$ & 6.43 \\
\hline & $2{ }^{1} \mathrm{~A}_{1}$ & $\pi \rightarrow \pi^{*}$ & 6.57 & 6.79 & 6.76 & $6.61(84.9)$ & 6.52 \\
\hline & $3{ }^{1} \mathrm{~A}_{1}$ & $\pi \rightarrow \pi^{*}$ & 8.74 & 9.19 & 10.09 & $8.53(90.7)$ & 8.22 \\
\hline pyrrole & $2{ }^{1} \mathrm{~A}_{1}$ & $\pi \rightarrow \pi^{*}$ & 6.18 & 6.44 & 6.52 & $6.40(86.0)$ & 6.31 \\
\hline
\end{tabular}




\begin{tabular}{|c|c|c|c|c|c|c|c|}
\hline Molecule & State & Type & MC-RPA & MC-TDA & SA-CAS & $\mathrm{CC}^{\mathrm{a}, \mathrm{b}}\left(\% \mathrm{~T}_{1}\right)$ & $\mathrm{CASPT}^{\mathrm{a}, \mathrm{c}}$ \\
\hline & $1^{1} \mathrm{~B}_{2}$ & $\pi \rightarrow \pi^{*}$ & 7.08 & 7.35 & 7.63 & $6.71(91.6)$ & 6.33 \\
\hline & $3{ }^{1} \mathrm{~A}_{1}$ & $\pi \rightarrow \pi^{*}$ & 8.16 & 8.68 & 9.52 & $8.17(90.2)$ & 8.17 \\
\hline \multirow[t]{5}{*}{ imidazole } & $1^{1} \mathrm{~A} "$ & $n \rightarrow \pi^{*}$ & 7.00 & 7.59 & 6.20 & $6.82(87.6)$ & 6.81 \\
\hline & $2{ }^{1} \mathrm{~A}^{\prime}$ & $\pi \rightarrow \pi^{*}$ & 6.47 & 6.77 & 7.49 & $6.58(87.2)$ & 6.19 \\
\hline & $3{ }^{1} \mathrm{~A}^{\prime}$ & $\pi \rightarrow \pi^{*}$ & 7.36 & 7.66 & 8.90 & $7.10(89.8)$ & 6.93 \\
\hline & $2{ }^{1} \mathrm{~A} "$ & $n \rightarrow \pi^{*}$ & 9.98 & 10.08 & 7.09 & $7.93(89.4)$ & 7.91 \\
\hline & $4^{1} \mathrm{~A}^{\prime}$ & $\pi \rightarrow \pi^{*}$ & 8.40 & 8.94 & 9.94 & $8.45(88.6)$ & 8.15 \\
\hline \multirow[t]{6}{*}{ pyridine } & $1^{1} \mathrm{~B}_{2}$ & $\pi \rightarrow \pi^{*}$ & 4.69 & 4.87 & 4.91 & $5.15(85.9)$ & 5.02 \\
\hline & $1^{1} \mathrm{~B}_{1}$ & $n \rightarrow \pi^{*}$ & 5.39 & 5.89 & 5.17 & $5.05(88.1)$ & 5.14 \\
\hline & $2^{1} \mathrm{~A}_{2}$ & $n \rightarrow \pi^{*}$ & 7.96 & 8.05 & 6.12 & $5.50(87.7)$ & 5.47 \\
\hline & $2{ }^{1} \mathrm{~A}_{1}$ & $\pi \rightarrow \pi^{*}$ & 7.42 & 7.68 & 8.40 & $6.85(92.8)$ & 6.39 \\
\hline & $3{ }^{1} \mathrm{~A}_{1}$ & $\pi \rightarrow \pi^{*}$ & 8.43 & 8.73 & 9.85 & $7.70(91.5)$ & 7.46 \\
\hline & $2{ }^{1} \mathrm{~B}_{2}$ & $\pi \rightarrow \pi^{*}$ & 8.00 & 8.25 & 8.22 & $7.59(89.7)$ & 7.29 \\
\hline \multirow[t]{7}{*}{ pyrazine } & $1{ }^{1} \mathrm{~B}_{3 \mathrm{u}}$ & $n \rightarrow \pi^{*}$ & 4.69 & 5.13 & 7.04 & $4.24(89.9)$ & 4.12 \\
\hline & $1^{1} \mathrm{~A}_{\mathrm{u}}$ & $n \rightarrow \pi^{*}$ & 7.45 & 7.53 & 8.14 & $5.05(88.4)$ & 4.70 \\
\hline & $1{ }^{1} \mathrm{~B}_{2 \mathrm{u}}$ & $\pi \rightarrow \pi^{*}$ & 4.55 & 4.73 & 4.82 & $5.02(86.2)$ & 4.85 \\
\hline & $1{ }^{1} \mathrm{~B}_{1 \mathrm{u}}$ & $\pi \rightarrow \pi^{*}$ & 7.75 & 8.02 & 8.68 & $7.07(93.3)$ & 6.89 \\
\hline & $2{ }^{1} \mathrm{~B}_{1 \mathrm{u}}$ & $\pi \rightarrow \pi^{*}$ & 8.87 & 9.22 & 8.68 & $8.06(90.9)$ & 7.79 \\
\hline & $2{ }^{1} \mathrm{~B}_{2 \mathrm{u}}$ & $\pi \rightarrow \pi^{*}$ & 8.76 & 9.02 & 9.99 & $8.05(89.7)$ & 7.65 \\
\hline & $2{ }^{1} \mathrm{~A}_{\mathrm{g}}$ & $\pi \rightarrow \pi^{*}$ & 8.24 & 8.48 & 8.24 & $8.69(74.2)$ & 8.61 \\
\hline \multirow[t]{6}{*}{ pyrimidine } & $1^{1} \mathrm{~B}_{1}$ & $n \rightarrow \pi^{*}$ & 5.17 & 5.69 & 4.83 & $4.50(88.4)$ & 4.44 \\
\hline & $1^{1} \mathrm{~A}_{2}$ & $n \rightarrow \pi^{*}$ & 5.92 & 6.40 & 5.54 & $4.93(88.2)$ & 4.81 \\
\hline & $1^{1} \mathrm{~B}_{2}$ & $\pi \rightarrow \pi^{*}$ & 4.84 & 5.04 & 5.07 & $5.36(85.7)$ & 5.24 \\
\hline & $2{ }^{1} \mathrm{~A}_{1}$ & $\pi \rightarrow \pi^{*}$ & 7.54 & 7.81 & 8.29 & $7.06(92.2)$ & 6.64 \\
\hline & $2{ }^{1} \mathrm{~B}_{2}$ & $\pi \rightarrow \pi^{*}$ & 8.75 & 9.07 & 10.05 & $8.01(90.7)$ & 7.64 \\
\hline & $3{ }^{1} \mathrm{~A}_{1}$ & $\pi \rightarrow \pi^{*}$ & 8.11 & 8.39 & 10.02 & $7.74(89.7)$ & 7.21 \\
\hline \multirow[t]{8}{*}{ pyridazine } & ${ }^{1}{ }^{1} \mathrm{~B}_{1}$ & $n \rightarrow \pi^{*}$ & 4.56 & 4.95 & 4.21 & $3.92(89.0)$ & 3.78 \\
\hline & $1^{1} \mathrm{~A}_{2}$ & $n \rightarrow \pi^{*}$ & 5.14 & 5.77 & 6.03 & $4.49(86.6)$ & 4.32 \\
\hline & $2{ }^{1} \mathrm{~A}_{1}$ & $\pi \rightarrow \pi^{*}$ & 4.68 & 4.87 & 4.89 & $5.22(85.2)$ & 5.18 \\
\hline & $2^{1} \mathrm{~A}_{2}$ & $n \rightarrow \pi^{*}$ & 7.85 & 7.97 & 4.89 & $5.74(86.6)$ & 5.77 \\
\hline & $2{ }^{1} \mathrm{~B}_{1}$ & $n \rightarrow \pi^{*}$ & 8.67 & 8.81 & 6.96 & $6.41(86.6)$ & 6.52 \\
\hline & ${ }^{1}{ }^{1} \mathrm{~B}_{2}$ & $\pi \rightarrow \pi^{*}$ & 7.51 & 7.75 & 8.77 & $6.93(90.7)$ & 6.31 \\
\hline & $2{ }^{1} \mathrm{~B}_{2}$ & $\pi \rightarrow \pi^{*}$ & 8.48 & 8.76 & 9.73 & $7.55(90.2)$ & 7.29 \\
\hline & $3{ }^{1} \mathrm{~A}_{1}$ & $\pi \rightarrow \pi^{*}$ & 8.30 & 8.58 & 10.18 & $7.82(90.5)$ & 7.62 \\
\hline \multirow[t]{6}{*}{$s$-triazine } & $1^{1} \mathrm{~A}_{1} "$ & $n \rightarrow \pi^{*}$ & 5.25 & 5.85 & 5.05 & $4.78(88.0)$ & 4.60 \\
\hline & $1^{1} \mathrm{~A}_{2} "$ & $n \rightarrow \pi^{*}$ & 7.45 & 7.53 & 5.84 & $4.76(88.0)$ & 5.79 \\
\hline & $1^{1} \mathrm{E} "$ & $n \rightarrow \pi^{*}$ & 5.91 & 6.38 & 5.44 & $4.81(88.1)$ & 4.71 \\
\hline & $1^{1} \mathrm{~A}_{2}$ & $\pi \rightarrow \pi^{*}$ & 5.09 & 5.32 & 5.29 & $5.71(85.1)$ & 4.68 \\
\hline & $2{ }^{1} \mathrm{~A}_{1}$ & $\pi \rightarrow \pi^{*}$ & 7.66 & 8.01 & 8.89 & $7.41(90.8)$ & 7.25 \\
\hline & $2{ }^{1} \mathrm{E} "$ & $n \rightarrow \pi^{*}$ & 9.45 & 9.70 & 9.41 & $7.80(88.1)$ & 7.72 \\
\hline
\end{tabular}




\begin{tabular}{|c|c|c|c|c|c|c|c|}
\hline Molecule & State & Type & MC-RPA & MC-TDA & SA-CAS & $\mathrm{CC}^{\mathrm{a}, \mathrm{b}}\left(\% \mathrm{~T}_{1}\right)$ & CASPT2 $^{\mathrm{a}, \mathrm{c}}$ \\
\hline & $1^{1} \mathrm{E}^{\prime}$ & $\pi \rightarrow \pi^{*}$ & 8.44 & 8.70 & 10.53 & $8.04(88.8)$ & 7.49 \\
\hline \multirow[t]{11}{*}{$s$-tetrazine } & $1^{1} \mathrm{~B}_{3 \mathrm{u}}$ & $n \rightarrow \pi^{*}$ & 3.37 & 3.74 & 3.14 & $2.53(89.6)$ & 2.24 \\
\hline & $1{ }^{1} \mathrm{~A}_{\mathrm{u}}$ & $n \rightarrow \pi^{*}$ & 5.09 & 5.57 & 4.56 & $3.79(87.5)$ & 3.48 \\
\hline & $1{ }^{1} \mathrm{~B}_{1 \mathrm{~g}}$ & $n \rightarrow \pi^{*}$ & 5.47 & 5.82 & 5.39 & $4.97(82.5)$ & 4.73 \\
\hline & $1{ }^{1} \mathrm{~B}_{2 \mathrm{u}}$ & $\pi \rightarrow \pi^{*}$ & 4.49 & 4.69 & 4.84 & $5.12(84.6)$ & 4.91 \\
\hline & $1{ }^{1} \mathrm{~B}_{2 \mathrm{~g}}$ & $n \rightarrow \pi^{*}$ & 5.09 & 5.91 & 5.20 & $5.34(80.7)$ & 5.18 \\
\hline & $2{ }^{1} \mathrm{~A}_{\mathrm{u}}$ & $n \rightarrow \pi^{*}$ & 7.20 & 7.37 & 6.37 & $5.46(87.4)$ & 5.47 \\
\hline & $2{ }^{1} \mathrm{~B}_{2 \mathrm{~g}}$ & $n \rightarrow \pi^{*}$ & 9.80 & 9.86 & 6.77 & $6.23(79.2)$ & 6.07 \\
\hline & $2{ }^{1} \mathrm{~B}_{1 \mathrm{~g}}$ & $n \rightarrow \pi^{*}$ & 10.18 & 10.34 & 7.48 & $6.87(84.7)$ & 6.38 \\
\hline & $2{ }^{1} \mathrm{~B}_{3 \mathrm{u}}$ & $n \rightarrow \pi^{*}$ & 8.88 & 9.03 & 7.88 & $6.67(86.7)$ & 6.77 \\
\hline & $1{ }^{1} \mathrm{~B}_{1 \mathrm{u}}$ & $\pi \rightarrow \pi^{*}$ & 8.38 & 8.71 & 9.53 & $7.45(91.0)$ & 6.96 \\
\hline & $2{ }^{1} \mathrm{~B}_{1 \mathrm{u}}$ & $\pi \rightarrow \pi^{*}$ & 8.84 & 9.12 & 10.29 & $7.79(90.2)$ & 7.43 \\
\hline \multirow[t]{3}{*}{ formaldehyde } & $1^{1} \mathrm{~A}_{2}$ & $n \rightarrow \pi^{*}$ & 3.83 & 4.32 & 3.77 & $3.95(91.2)$ & 3.98 \\
\hline & $1^{1} \mathrm{~B}_{1}$ & $\sigma \rightarrow \pi^{*}$ & 8.99 & 9.54 & 9.04 & $9.18(90.9)$ & 9.14 \\
\hline & $2{ }^{1} \mathrm{~A}_{1}$ & $\pi \rightarrow \pi^{*}$ & 9.25 & 10.23 & 12.07 & $10.45(91.3)$ & 9.31 \\
\hline \multirow[t]{3}{*}{ acetone } & $1^{1} \mathrm{~A}_{2}$ & $n \rightarrow \pi^{*}$ & 4.35 & 4.91 & 3.98 & $4.40(90.8)$ & 4.42 \\
\hline & $1^{1} \mathrm{~B}_{1}$ & $\sigma \rightarrow \pi^{*}$ & 8.99 & 9.77 & 10.07 & $9.17(91.5)$ & 9.27 \\
\hline & $2{ }^{1} \mathrm{~A}_{1}$ & $\pi \rightarrow \pi^{*}$ & 9.25 & 9.78 & 11.73 & $9.65(90.1)$ & 9.31 \\
\hline \multirow{4}{*}{$\begin{array}{l}p \text { - } \\
\text { benzoquinone }\end{array}$} & $1^{1} \mathrm{~A}_{\mathrm{u}}$ & $n \rightarrow \pi^{*}$ & 3.46 & 4.00 & 3.13 & $2.85(83.0)$ & 2.80 \\
\hline & $1{ }^{1} \mathrm{~B}_{1 \mathrm{~g}}$ & $n \rightarrow \pi^{*}$ & 3.41 & 3.92 & 3.14 & $2.75(84.1)$ & 2.78 \\
\hline & $1{ }^{1} \mathrm{~B}_{3 \mathrm{~g}}$ & $\pi \rightarrow \pi^{*}$ & 5.92 & 6.01 & 5.92 & $4.59(87.9)$ & 4.25 \\
\hline & $1{ }^{1} \mathrm{~B}_{1 \mathrm{u}}$ & $\pi \rightarrow \pi^{*}$ & 6.71 & 7.04 & 7.71 & $5.62(88.4)$ & 5.29 \\
\hline \multirow[t]{3}{*}{ formamide } & $1^{1} \mathrm{~A} "$ & $n \rightarrow \pi^{*}$ & 5.46 & 6.22 & 5.21 & $5.65(90.7)$ & 5.63 \\
\hline & $2{ }^{1} \mathrm{~A}^{\prime}$ & $\pi \rightarrow \pi^{*}$ & 8.07 & 8.53 & 9.24 & $8.27(87.9)$ & 7.44 \\
\hline & $3{ }^{1} \mathrm{~A}^{\prime}$ & $\pi \rightarrow \pi^{*}$ & 11.28 & 11.57 & 12.19 & $10.93(86.6)$ & 10.54 \\
\hline \multirow[t]{3}{*}{ acetamide } & $1^{1} \mathrm{~A}^{\prime \prime}$ & $n \rightarrow \pi^{*}$ & 5.62 & 6.37 & 5.26 & $5.69(90.6)$ & 5.80 \\
\hline & $2{ }^{1} \mathrm{~A}^{\prime}$ & $\pi \rightarrow \pi^{*}$ & 8.17 & 8.62 & 9.25 & $7.67(89.1)$ & 7.27 \\
\hline & $3{ }^{1} \mathrm{~A}^{\prime}$ & $\pi \rightarrow \pi^{*}$ & 11.11 & 11.39 & 12.10 & $10.50(88.7)$ & 10.09 \\
\hline \multirow[t]{3}{*}{ propanamide } & $1^{1} \mathrm{~A}^{\prime \prime}$ & $n \rightarrow \pi^{*}$ & 5.66 & 6.41 & 5.27 & $5.72(90.6)$ & 5.72 \\
\hline & $2{ }^{1} \mathrm{~A}^{\prime}$ & $\pi \rightarrow \pi^{*}$ & 8.15 & 8.60 & 9.23 & $7.62(89.2)$ & 7.20 \\
\hline & $3{ }^{1} \mathrm{~A}^{\prime}$ & $\pi \rightarrow \pi^{*}$ & 10.97 & 11.14 & 12.03 & $10.06(89.0)$ & 9.94 \\
\hline \multirow[t]{5}{*}{ cytosine } & $2{ }^{1} \mathrm{~A}^{\prime}$ & $\pi \rightarrow \pi^{*}$ & 5.11 & 5.41 & 5.00 & $4.72(86)$ & 4.68 \\
\hline & $1^{1} \mathrm{~A} "$ & $n \rightarrow \pi^{*}$ & 5.68 & 6.42 & 5.01 & $5.16(86)$ & 5.12 \\
\hline & $2{ }^{1} \mathrm{~A} "$ & $n \rightarrow \pi^{*}$ & 5.83 & 6.73 & 5.34 & $5.52(83)$ & 5.54 \\
\hline & $3{ }^{1} \mathrm{~A}^{\prime}$ & $\pi \rightarrow \pi^{*}$ & 6.25 & 6.56 & 6.56 & $5.61(85)$ & 5.54 \\
\hline & $4{ }^{1} \mathrm{~A}^{\prime}$ & $\pi \rightarrow \pi^{*}$ & 7.11 & 7.49 & 8.18 & $6.61(88)$ & 6.40 \\
\hline \multirow[t]{2}{*}{ thymine } & $1^{1} \mathrm{~A} "$ & $n \rightarrow \pi^{*}$ & 5.17 & 5.94 & 4.88 & $4.98(87)$ & 4.94 \\
\hline & $2{ }^{1} \mathrm{~A}^{\prime}$ & $\pi \rightarrow \pi^{*}$ & 6.27 & 6.52 & 6.58 & $5.34(89)$ & 5.06 \\
\hline
\end{tabular}




\begin{tabular}{lllrrrrr}
\hline Molecule & State & Type & MC-RPA & MC-TDA & SA-CAS & CC3 $^{\mathrm{a}, \mathrm{b}}\left(\% \mathrm{~T}_{1}\right)$ & CASPT2 $^{\mathrm{a}, \mathrm{c}}$ \\
\hline & $3{ }^{1} \mathrm{~A}^{\prime}$ & $\pi \rightarrow \pi^{*}$ & 7.34 & 7.64 & 7.10 & $6.34(83)$ & 6.15 \\
& $2{ }^{1} \mathrm{~A}^{\prime}$ & $n \rightarrow \pi^{*}$ & 6.50 & 7.44 & 6.44 & $6.45(89)$ & 6.38 \\
& $4^{1} \mathrm{~A}^{\prime}$ & $\pi \rightarrow \pi^{*}$ & 7.88 & 8.18 & 8.19 & $6.71(88)$ & 6.52 \\
uracil & $1^{1} \mathrm{~A}^{\prime \prime}$ & $n \rightarrow \pi^{*}$ & 5.14 & 5.91 & 4.90 & $4.90(86)$ & 4.90 \\
& $2{ }^{1} \mathrm{~A}^{\prime}$ & $\pi \rightarrow \pi^{*}$ & 6.25 & 6.52 & 6.51 & $5.44(88)$ & 5.23 \\
& $3{ }^{1} \mathrm{~A}^{\prime}$ & $\pi \rightarrow \pi^{*}$ & 7.35 & 7.62 & 6.97 & $6.29(83)$ & 6.15 \\
& $2{ }^{1} \mathrm{~A}^{\prime \prime}$ & $n \rightarrow \pi^{*}$ & 6.46 & 7.39 & 6.46 & $6.32(88)$ & 6.27 \\
& $4{ }^{1} \mathrm{~A}^{\prime}$ & $\pi \rightarrow \pi^{*}$ & 7.99 & 8.30 & 8.64 & $6.84(88)$ & 6.75 \\
& $2{ }^{1} \mathrm{~A}^{\prime}$ & $\pi \rightarrow \pi^{*}$ & 4.96 & 5.23 & 5.22 & $5.18(86)$ & 5.20 \\
& $3{ }^{1} \mathrm{~A}^{\prime}$ & $\pi \rightarrow \pi^{*}$ & 5.96 & 6.24 & 6.63 & $5.39(89)$ & 5.30 \\
& $1^{1} \mathrm{~A}^{\prime \prime}$ & $n \rightarrow \pi^{*}$ & 6.39 & 6.92 & 5.96 & $5.34(88)$ & 5.21 \\
& $2{ }^{1} \mathrm{~A}^{\prime \prime}$ & $n \rightarrow \pi^{*}$ & 6.63 & 7.23 & 6.48 & $5.96(88)$ & 5.97 \\
& $4{ }^{1} \mathrm{~A}^{\prime}$ & $\pi \rightarrow \pi^{*}$ & 7.06 & 7.34 & 7.64 & $6.53(87)$ & 6.35 \\
& & & & & & & \\
\hline
\end{tabular}

aTaken from Ref. 1.

${ }^{\mathrm{b}}$ Taken from Ref. 2.

${ }^{\mathrm{c}} \mathrm{MS}-\mathrm{CASPT} 2$ on top of SA-CASSCF calculation.

${ }^{\mathrm{d}}$ Subscript labels of B species are inline with IUPAC convention[3] but deviate from Ref. 1 . 


\section{Vertical excitation energies with aug-cc-pVTZ basis set}

Table 4: Vertical singlet excitation energies in eV with aug-cc-pVTZ basis set.

\begin{tabular}{lllrrrrr}
\hline Molecule & State & Type & MC-RPA & MC-TDA & SA-CAS & CC $^{\mathrm{a}}$ & CASPT2 $^{\mathrm{b}, \mathrm{c}}$ \\
\hline ethene & $1{ }^{1} \mathrm{~B}_{1 \mathrm{u}}$ & $\pi \rightarrow \pi^{*}$ & 8.33 & 8.57 & 7.74 & 7.89 & 7.84 \\
cyclopropene & $2^{1} \mathrm{~A}_{\mathrm{g}}$ & $\pi \rightarrow \pi^{*}$ & 6.91 & 6.93 & 6.59 & 6.63 & 6.43 \\
& $1{ }^{1} \mathrm{~B}_{1}$ & $\sigma \rightarrow \pi^{*}$ & 7.44 & 7.53 & 7.02 & 6.68 & 6.63 \\
benzene & $1{ }^{1} \mathrm{~B}_{2}$ & $\pi \rightarrow \pi^{*}$ & 7.30 & 7.55 & 7.63 & 6.67 & 6.66 \\
& $1{ }^{1} \mathrm{~B}_{2 \mathrm{u}}$ & $\pi \rightarrow \pi^{*}$ & 4.63 & 4.80 & 4.81 & 5.03 & 4.96 \\
& $1{ }^{1} \mathrm{~B}_{1 \mathrm{u}}$ & $\pi \rightarrow \pi^{*}$ & 7.09 & 7.34 & 7.78 & 6.42 & 6.57 \\
& $1{ }^{1} \mathrm{E}_{1 \mathrm{u}}$ & $\pi \rightarrow \pi^{*}$ & 7.85 & 8.07 & 9.17 & 7.14 & 7.36 \\
\multirow{5}{*}{-tetrazine } & $2{ }^{1} \mathrm{E}_{2 \mathrm{~g}}$ & $\pi \rightarrow \pi^{*}$ & 8.03 & 8.34 & 8.01 & 8.31 & 8.15 \\
& $1{ }^{1} \mathrm{~B}_{3 \mathrm{u}}$ & $n \rightarrow \pi^{*}$ & 3.36 & 3.75 & 3.09 & 2.46 & 2.27 \\
& $1{ }^{1} \mathrm{~B}_{\mathrm{g}}$ & $n \rightarrow \pi^{*}$ & 5.49 & 5.87 & 5.36 & 4.87 & 4.74 \\
& $1{ }^{1} \mathrm{~B}_{2 \mathrm{u}}$ & $\pi \rightarrow \pi^{*}$ & 4.46 & 4.67 & 4.83 & 5.08 & 4.89 \\
& $1{ }^{1} \mathrm{~B}_{2 \mathrm{~g}}$ & $n \rightarrow \pi^{*}$ & 5.13 & 5.98 & 5.18 & 5.28 & 5.07 \\
& $2{ }^{1} \mathrm{~B}_{2 \mathrm{~g}}$ & $n \rightarrow \pi^{*}$ & 9.65 & 9.72 & 6.75 & 6.16 & 5.84 \\
& $2{ }^{1} \mathrm{~B}_{3 \mathrm{u}}$ & $n \rightarrow \pi^{*}$ & 9.94 & $/ \mathrm{d}$ & 7.44 & 6.80 & 6.33 \\
& $1{ }^{1} \mathrm{~B}_{1 \mathrm{u}}$ & $n \rightarrow \pi^{*}$ & 8.80 & 8.96 & 7.83 & 6.60 & 6.59 \\
& $2{ }^{1} \mathrm{~B}_{1 \mathrm{u}}$ & $\pi \rightarrow \pi^{*}$ & 8.17 & 8.51 & 9.37 & 7.18 & 6.84 \\
& $2{ }^{1} \mathrm{~B}_{3 \mathrm{~g}}$ & $\pi \rightarrow \pi^{*}$ & 8.67 & 8.94 & 10.18 & 7.59 & 7.20 \\
formamide & $1{ }^{1} \mathrm{~A}^{*}$ & $n \rightarrow \pi^{*}$ & 5.56 & 6.32 & 5.08 & 5.55 & 5.58 \\
\hline & $1{ }^{1} \mathrm{~A}_{2}$ & $n \rightarrow \pi^{*}$ & 3.89 & 4.41 & 3.88 & 3.88 & 4.01 \\
& $1{ }^{1} \mathrm{~B}_{1}$ & $\sigma \rightarrow \pi^{*}$ & 8.63 & 9.62 & 9.15 & 9.05 & 9.12 \\
& $1{ }^{1} \mathrm{~B}_{1}$ & $\sigma \rightarrow \pi^{*}$ & 9.35 & 9.93 & 11.71 & 9.31 & 9.47 \\
& $1{ }^{1} \mathrm{~A}_{2}$ & $n \rightarrow \pi^{*}$ & 4.45 & 5.02 & 4.25 & 4.38 & 4.49 \\
& $2{ }^{1} \mathrm{~A}_{1}$ & $\pi \rightarrow \pi^{*}$ & 8.93 & 9.35 & 10.97 & 8.90 & 9.19 \\
& & & & & & &
\end{tabular}

ataken from Ref. 4.

${ }^{\mathrm{b}}$ Taken from Ref. 5.

${ }^{\mathrm{c}}$ MS-CASPT2 on top of SA-CASSCF calculation.

${ }^{\mathrm{d}}$ Not match found for the 70 lowest MC-TDA states. 


\section{Oscillator strength with TZVP basis set}

Table 5: Oscillator strengths with TZVP basis set for allowed vertical singlet transitions given in atomic units.

\begin{tabular}{|c|c|c|c|c|c|c|c|}
\hline Molecule & State & Type & MC-RPA & MC-TDA & SA-CAS & $\mathrm{CC} 3^{\mathrm{a}}$ & CASPT2 $^{\mathrm{b}}$ \\
\hline ethene & $1^{1} \mathrm{~B}_{1 \mathrm{u}}$ & $\pi \rightarrow \pi^{*}$ & 0.417 & 0.440 & 0.611 & 0.389 & 0.513 \\
\hline E-butadiene & $1^{1} \mathrm{~B}_{\mathrm{u}}$ & $\pi \rightarrow \pi^{*}$ & 0.814 & 0.839 & 1.029 & 0.726 & 0.783 \\
\hline $\begin{array}{l}\text { all-E- } \\
\text { hexatriene }\end{array}$ & $1{ }^{1} \mathrm{~B}_{\mathrm{u}}$ & $\pi \rightarrow \pi^{*}$ & 1.230 & 1.230 & 1.449 & 1.129 & 1.045 \\
\hline $\begin{array}{l}\text { all-E- } \\
\text { octatetraene }\end{array}$ & $1^{1} \mathrm{~B}_{\mathrm{u}}$ & $\pi \rightarrow \pi^{*}$ & 1.629 & 1.520 & 1.849 & 1.549 & 1.299 \\
\hline \multirow[t]{2}{*}{ cyclopropene } & ${ }^{1}{ }^{1} \mathrm{~B}_{1}$ & $\sigma \rightarrow \pi^{*}$ & 0.001 & 0.001 & 0.007 & 0.001 & 0.001 \\
\hline & $1{ }^{1} \mathrm{~B}_{2}$ & $\pi \rightarrow \pi^{*}$ & 0.095 & 0.096 & 0.346 & 0.083 & 0.234 \\
\hline \multirow[t]{3}{*}{ cyclopentadiene } & $1^{1} \mathrm{~B}_{2}$ & $\pi \rightarrow \pi^{*}$ & 0.085 & 0.101 & 0.233 & 0.093 & 0.144 \\
\hline & $2{ }^{1} \mathrm{~A}_{1}$ & $\pi \rightarrow \pi^{*}$ & 0.001 & 0.001 & 0.005 & 0.005 & 0.001 \\
\hline & $3{ }^{1} \mathrm{~A}_{1}$ & $\pi \rightarrow \pi^{*}$ & 0.679 & 0.749 & 0.575 & 0.596 & 0.965 \\
\hline \multirow[t]{2}{*}{ norbornadiene } & $1^{1} \mathrm{~B}_{2}$ & $\pi \rightarrow \pi^{*}$ & 0.154 & 0.126 & 0.093 & 0.027 & 0.092 \\
\hline & $2{ }^{1} \mathrm{~B}_{2}$ & $\pi \rightarrow \pi^{*}$ & 0.118 & 0.153 & 0.816 & 0.185 & 0.393 \\
\hline benzene & $1{ }^{1} \mathrm{E}_{1 \mathrm{u}}$ & $\pi \rightarrow \pi^{*}$ & 0.675 & 0.755 & 1.115 & 0.630 & 0.847 \\
\hline \multirow[t]{3}{*}{ naphthalene } & $1^{1} \mathrm{~B}_{1 \mathrm{u}}$ & $\pi \rightarrow \pi^{*}$ & 0.100 & 0.101 & 0.104 & 0.085 & 0.137 \\
\hline & $2{ }^{1} \mathrm{~B}_{2 \mathrm{u}}$ & $\pi \rightarrow \pi^{*}$ & 1.435 & 1.561 & 2.030 & 1.325 & 1.548 \\
\hline & $2{ }^{1} \mathrm{~B}_{1 \mathrm{u}}$ & $\pi \rightarrow \pi^{*}$ & 0.311 & 0.341 & 0.384 & 0.239 & 0.402 \\
\hline \multirow[t]{3}{*}{ furan } & $1^{1} \mathrm{~B}_{2}$ & $\pi \rightarrow \pi^{*}$ & 0.134 & 0.158 & 0.263 & 0.155 & 0.199 \\
\hline & $2{ }^{1} \mathrm{~A}_{1}$ & $\pi \rightarrow \pi^{*}$ & 0.001 & 0.001 & 0.004 & 0.001 & 0.008 \\
\hline & $3{ }^{1} \mathrm{~A}_{1}$ & $\pi \rightarrow \pi^{*}$ & 0.440 & 0.511 & 0.637 & 0.450 & 0.793 \\
\hline \multirow[t]{3}{*}{ pyrrole } & $1^{1} \mathrm{~B}_{2}$ & $\pi \rightarrow \pi^{*}$ & 0.147 & 0.164 & 0.223 & 0.167 & 0.199 \\
\hline & $2{ }^{1} \mathrm{~A}_{1}$ & $\pi \rightarrow \pi^{*}$ & 0.008 & 0.005 & 0.007 & 0.004 & 0.008 \\
\hline & $3{ }^{1} \mathrm{~A}_{1}$ & $\pi \rightarrow \pi^{*}$ & 0.459 & 0.556 & 0.761 & 0.478 & 0.793 \\
\hline \multirow[t]{5}{*}{ imidazole } & $1^{1} \mathrm{~A}^{\prime \prime}$ & $n \rightarrow \pi^{*}$ & 0.010 & 0.012 & 0.003 & 0.004 & 0.010 \\
\hline & $2{ }^{1} \mathrm{~A}^{\prime}$ & $\pi \rightarrow \pi^{*}$ & 0.019 & 0.025 & 0.038 & 0.081 & 0.229 \\
\hline & $3{ }^{1} \mathrm{~A}^{\prime}$ & $\pi \rightarrow \pi^{*}$ & 0.132 & 0.148 & 0.292 & 0.082 & 0.062 \\
\hline & $2{ }^{1} \mathrm{~A} "$ & $n \rightarrow \pi^{*}$ & 0.001 & 0.001 & 0.049 & 0.005 & 0.019 \\
\hline & $4^{1} \mathrm{~A}^{\prime}$ & $\pi \rightarrow \pi^{*}$ & 0.406 & 0.463 & 0.595 & 0.410 & 0.555 \\
\hline \multirow[t]{5}{*}{ pyridine } & ${ }^{1}{ }^{1} \mathrm{~B}_{2}$ & $\pi \rightarrow \pi^{*}$ & 0.013 & 0.013 & 0.004 & 0.021 & 0.044 \\
\hline & $1^{1} \mathrm{~B}_{1}$ & $n \rightarrow \pi^{*}$ & 0.008 & 0.010 & 0.009 & 0.005 & 0.009 \\
\hline & $2{ }^{1} \mathrm{~A}_{1}$ & $\pi \rightarrow \pi^{*}$ & 0.010 & 0.013 & 0.017 & 0.014 & 0.004 \\
\hline & $3{ }^{1} \mathrm{~A}_{1}$ & $\pi \rightarrow \pi^{*}$ & 0.535 & 0.609 & 1.090 & 0.526 & 0.849 \\
\hline & $2{ }^{1} \mathrm{~B}_{2}$ & $\pi \rightarrow \pi^{*}$ & 0.264 & 0.296 & 0.022 & 0.482 & 0.691 \\
\hline
\end{tabular}




\begin{tabular}{|c|c|c|c|c|c|c|c|}
\hline Molecule & State & Type & MC-RPA & MC-TDA & SA-CAS & $\mathrm{CC}^{\mathrm{a}}$ & CASPT2 $^{b}$ \\
\hline \multirow[t]{5}{*}{ pyrazine } & $1{ }^{1} \mathrm{~B}_{3 \mathrm{u}}$ & $n \rightarrow \pi^{*}$ & 0.012 & 0.014 & 0.021 & 0.007 & 0.012 \\
\hline & $1^{1} \mathrm{~B}_{2 \mathrm{u}}$ & $\pi \rightarrow \pi^{*}$ & 0.042 & 0.044 & 0.038 & 0.062 & 0.123 \\
\hline & $1^{1} \mathrm{~B}_{1 \mathrm{u}}$ & $\pi \rightarrow \pi^{*}$ & 0.077 & 0.094 & 0.084 & 0.070 & 0.107 \\
\hline & $2{ }^{1} \mathrm{~B}_{1 \mathrm{u}}$ & $\pi \rightarrow \pi^{*}$ & 0.478 & 0.522 & 1.024 & 0.407 & 0.774 \\
\hline & $2{ }^{1} \mathrm{~B}_{2 \mathrm{u}}$ & $\pi \rightarrow \pi^{*}$ & 0.479 & 0.541 & 0.959 & 0.376 & 0.622 \\
\hline \multirow[t]{5}{*}{ pyrimidine } & ${ }^{1}{ }^{1} \mathrm{~B}_{1}$ & $n \rightarrow \pi^{*}$ & 0.015 & 0.018 & 0.015 & 0.006 & 0.013 \\
\hline & $1^{1} \mathrm{~B}_{2}$ & $\pi \rightarrow \pi^{*}$ & 0.012 & 0.013 & 0.007 & 0.021 & 0.049 \\
\hline & $2{ }^{1} \mathrm{~A}_{1}$ & $\pi \rightarrow \pi^{*}$ & 0.030 & 0.048 & 0.066 & 0.043 & 0.164 \\
\hline & $3^{1} \mathrm{~A}_{1}$ & $\pi \rightarrow \pi^{*}$ & 0.242 & 0.244 & 0.962 & 0.391 & 0.540 \\
\hline & $2{ }^{1} \mathrm{~B}_{2}$ & $\pi \rightarrow \pi^{*}$ & 0.456 & 0.533 & 0.869 & 0.415 & 0.768 \\
\hline \multirow[t]{6}{*}{ pyridazine } & ${ }^{1}{ }^{1} \mathrm{~B}_{1}$ & $n \rightarrow \pi^{*}$ & 0.013 & 0.017 & 0.013 & 0.006 & 0.010 \\
\hline & $2^{1} \mathrm{~A}_{1}$ & $\pi \rightarrow \pi^{*}$ & 0.009 & 0.009 & 0.005 & 0.014 & 0.027 \\
\hline & $2^{1} \mathrm{~B}_{1}$ & $n \rightarrow \pi^{*}$ & 0.004 & 0.005 & 0.005 & 0.005 & 0.008 \\
\hline & $1^{1} \mathrm{~B}_{2}$ & $\pi \rightarrow \pi^{*}$ & 0.033 & 0.032 & 0.002 & 0.012 & 0.115 \\
\hline & $2{ }^{1} \mathrm{~B}_{2}$ & $\pi \rightarrow \pi^{*}$ & 0.403 & 0.442 & 0.966 & 0.340 & 0.603 \\
\hline & $3{ }^{1} \mathrm{~A}_{1}$ & $\pi \rightarrow \pi^{*}$ & 0.194 & 0.187 & 1.075 & 0.433 & 0.742 \\
\hline \multirow[t]{2}{*}{$s$-triazine } & $1^{1} \mathrm{~A}_{2}{ }^{\prime \prime}$ & $n \rightarrow \pi^{*}$ & 0.027 & 0.034 & 0.038 & 0.016 & 0.021 \\
\hline & $1{ }^{1} \mathrm{E}^{\prime}$ & $\pi \rightarrow \pi^{*}$ & 0.258 & 0.263 & 0.912 & 0.386 & 0.680 \\
\hline \multirow[t]{5}{*}{$s$-tetrazine } & $1^{1} \mathrm{~B}_{3 \mathrm{u}}$ & $n \rightarrow \pi^{*}$ & 0.018 & 0.023 & 0.020 & 0.007 & 0.013 \\
\hline & $1^{1} \mathrm{~B}_{2 \mathrm{u}}$ & $\pi \rightarrow \pi^{*}$ & 0.029 & 0.029 & 0.024 & 0.044 & 0.110 \\
\hline & $2{ }^{1} \mathrm{~B}_{3 \mathrm{u}}$ & $n \rightarrow \pi^{*}$ & 0.015 & 0.020 & 0.025 & 0.011 & 0.021 \\
\hline & $1^{1} \mathrm{~B}_{1 \mathrm{u}}$ & $\pi \rightarrow \pi^{*}$ & 0.003 & 0.017 & 0.027 & 0.002 & 0.136 \\
\hline & $2{ }^{1} \mathrm{~B}_{1 \mathrm{u}}$ & $\pi \rightarrow \pi^{*}$ & 0.366 & 0.400 & 0.782 & 0.349 & 0.496 \\
\hline \multirow[t]{2}{*}{ formaldehyde } & $1^{1} \mathrm{~B}_{1}$ & $\sigma \rightarrow \pi^{*}$ & 0.005 & 0.004 & 0.012 & 0.003 & 0.013 \\
\hline & $1^{1} \mathrm{~A}_{1}$ & $\pi \rightarrow \pi^{*}$ & 0.209 & 0.211 & 0.500 & 0.348 & 0.451 \\
\hline acetone & $2{ }^{1} \mathrm{~A}_{1}$ & $\pi \rightarrow \pi^{*}$ & 0.304 & 0.315 & 0.457 & 0.245 & 0.358 \\
\hline \multirow{3}{*}{$\begin{array}{l}p \text { - } \\
\text { benzoquinone } \\
\text { formamide }\end{array}$} & $1{ }^{1} \mathrm{~B}_{1 \mathrm{u}}$ & $\pi \rightarrow \pi^{*}$ & 0.819 & 0.815 & 0.631 & 0.485 & 0.638 \\
\hline & $2^{1} \mathrm{~A}^{\prime}$ & $\pi \rightarrow \pi^{*}$ & 0.273 & 0.282 & 0.555 & 0.386 & 0.479 \\
\hline & $3{ }^{1} \mathrm{~A}^{\prime}$ & $\pi \rightarrow \pi^{*}$ & 0.129 & 0.050 & 0.200 & 0.110 & 0.163 \\
\hline \multirow[t]{2}{*}{ acetamide } & $2{ }^{1} \mathrm{~A}^{\prime}$ & $\pi \rightarrow \pi^{*}$ & 0.270 & 0.279 & 0.506 & 0.207 & 0.424 \\
\hline & $3{ }^{1} \mathrm{~A}^{\prime}$ & $\pi \rightarrow \pi^{*}$ & 0.187 & 0.205 & 0.300 & 0.263 & 0.263 \\
\hline \multirow[t]{2}{*}{ propanamide } & $2{ }^{1} \mathrm{~A}^{\prime}$ & $\pi \rightarrow \pi^{*}$ & 0.235 & 0.236 & 0.489 & 0.170 & 0.405 \\
\hline & $3{ }^{1} \mathrm{~A}^{\prime}$ & $\pi \rightarrow \pi^{*}$ & 0.177 & 0.159 & 0.311 & 0.136 & 0.275 \\
\hline \multirow[t]{5}{*}{ cytosine } & $2{ }^{1} \mathrm{~A}^{\prime}$ & $\pi \rightarrow \pi^{*}$ & 0.063 & 0.069 & 0.090 & 0.046 & 0.093 \\
\hline & $1^{1} \mathrm{~A}^{\prime \prime}$ & $n \rightarrow \pi^{*}$ & 0.004 & 0.004 & 0.001 & 0.001 & 0.003 \\
\hline & $3^{1} \mathrm{~A}^{\prime}$ & $\pi \rightarrow \pi^{*}$ & 0.228 & 0.211 & 0.012 & 0.130 & 0.352 \\
\hline & $4^{1} \mathrm{~A}^{\prime}$ & $\pi \rightarrow \pi^{*}$ & 0.650 & 0.742 & 0.630 & 0.520 & 0.623 \\
\hline & $2{ }^{1} \mathrm{~A}^{\prime \prime}$ & $n \rightarrow \pi^{*}$ & $<0.001$ & 0.001 & 0.004 & 0.001 & 0.002 \\
\hline
\end{tabular}




\begin{tabular}{lllrrrrr}
\hline Molecule & State & Type & MC-RPA & MC-TDA & SA-CAS & CC $^{\mathrm{a}}$ & CASPT2 $^{\mathrm{b}}$ \\
\hline thymine & $2^{1} \mathrm{~A}^{\prime}$ & $\pi \rightarrow \pi^{*}$ & 0.365 & 0.337 & 0.240 & 0.172 & 0.334 \\
& $3^{1} \mathrm{~A}^{\prime}$ & $\pi \rightarrow \pi^{*}$ & 0.070 & 0.099 & 0.085 & 0.072 & 0.067 \\
& $4^{1} \mathrm{~A}^{\prime}$ & $\pi \rightarrow \pi^{*}$ & 0.376 & 0.331 & 0.251 & 0.197 & 0.356 \\
uracil & $2^{1} \mathrm{~A}^{\prime}$ & $\pi \rightarrow \pi^{*}$ & 0.371 & 0.345 & 0.272 & 0.174 & 0.320 \\
& $3^{1} \mathrm{~A}^{\prime}$ & $\pi \rightarrow \pi^{*}$ & 0.061 & 0.071 & 0.065 & 0.046 & 0.047 \\
& $4^{1} \mathrm{~A}^{\prime}$ & $\pi \rightarrow \pi^{*}$ & 0.319 & 0.271 & 0.196 & 0.152 & 0.249 \\
adenine & $1^{1} \mathrm{~A}^{\prime \prime}$ & $n \rightarrow \pi^{*}$ & 0.006 & 0.004 & 0.004 & 0.001 & 0.001 \\
& $2^{1} \mathrm{~A}^{\prime \prime}$ & $n \rightarrow \pi^{*}$ & 0.004 & 0.005 & 0.009 & 0.002 & 0.002 \\
\hline
\end{tabular}

\section{References}

[1] Schreiber, M.; Silva-Junior, M. R.; Sauer, S. P. A.; Thiel, W. J. Chem. Phys. 2008, $128,134110$.

[2] Kánnár, D.; Szalay, P. G. J. Chem. Theory Comput. 2014, 10, 3757-3765.

[3] Schutte C. J., H.; Bertie J., E.; Bunker P., R.; Hougen J., T.; Mills I., M.; Watson J. K., G.; Winnewisser B., P. Pure Appl. Chem. 1997, 69, 1641.

[4] Silva-Junior, M. R.; Sauer, S. P.; Schreiber, M.; Thiel, W. Mol. Phys. 2010, 108, 453-465.

[5] Silva-Junior, M. R.; Schreiber, M.; Sauer, S. P. A.; Thiel, W. J. Chem. Phys. 2010, 133.

\footnotetext{
ataken from Ref. 2.

${ }^{\mathrm{b}}$ Taken from Ref. 1.
} 